ARTICLE

Received 7 Jan 2015 | Accepted 3 Feb 2015 | Published 11 Mar 2015

DOI: $10.1038 /$ ncomms 7499 OPEN

\title{
Silane-catalysed fast growth of large single-crystalline graphene on hexagonal boron nitride
}

Shujie Tang ${ }^{1,2}$, Haomin Wang ${ }^{1}$, Hui Shan Wang ${ }^{1,3}$, Qiujuan Sun ${ }^{1,3}$, Xiuyun Zhang ${ }^{4}$, Chunxiao Cong ${ }^{5}$, Hong Xie ${ }^{1}$, Xiaoyu Liu ${ }^{1}$, Xiaohao Zhou ${ }^{6}$, Fuqiang Huang ${ }^{7}$, Xiaoshuang Chen ${ }^{6}$, Ting Yu ${ }^{5}$, Feng Ding ${ }^{4}$, Xiaoming $\mathrm{Xie}^{1,8} \&$ Mianheng Jiang ${ }^{1,8}$

The direct growth of high-quality, large single-crystalline domains of graphene on a dielectric substrate is of vital importance for applications in electronics and optoelectronics. Traditionally, graphene domains grown on dielectrics are typically only $\sim 1 \mu \mathrm{m}$ with a growth rate of $\sim 1 \mathrm{~nm} \mathrm{~min}-1$ or less, the main reason is the lack of a catalyst. Here we show that silane, serving as a gaseous catalyst, is able to boost the graphene growth rate to $\sim 1 \mu \mathrm{m} \mathrm{min}^{-1}$, thereby promoting graphene domains up to $20 \mu \mathrm{m}$ in size to be synthesized via chemical vapour deposition (CVD) on hexagonal boron nitride ( $h$-BN). Hall measurements show that the mobility of the sample reaches $20,000 \mathrm{~cm}^{2} \mathrm{~V}^{-1} \mathrm{~s}^{-1}$ at room temperature, which is among the best for CVD-grown graphene. Combining the advantages of both catalytic CVD and the ultra-flat dielectric substrate, gaseous catalyst-assisted CVD paves the way for synthesizing high-quality graphene for device applications while avoiding the transfer process.

\footnotetext{
${ }^{1}$ State Key Laboratory of Functional Materials for Informatics, Shanghai Institute of Microsystem and Information Technology, Chinese Academy of Sciences, 865 Changning Road, Shanghai 200050, China. ${ }^{2}$ Graduate University of the Chinese Academy of Sciences, Beijing 100049 , China. ${ }^{3}$ School of Physics and Electronics, Central South University, Changsha 410083, China. ${ }^{4}$ Institute of Textiles and Clothing, Hong Kong Polytechnic University, Kowloon, Hong Kong 999077, China. ${ }^{5}$ Division of Physics and Applied Physics, School of Physical and Mathematical Sciences, Nanyang Technological University, 21 Nanyang Link, Singapore 637371, Singapore. ${ }^{6}$ National Laboratory for Infrared Physics, Shanghai Institute of Technical Physics, Chinese Academy of Sciences, Shanghai 200083, China. ${ }^{7}$ CAS Key Laboratory of Materials for Energy Conversion, Shanghai Institute of Ceramics, Chinese Academy of Sciences, Shanghai 200050, China. ${ }^{8}$ School of Physical Science and Technology, ShanghaiTech University, 319 Yueyang Road, Shanghai 200031, China. Correspondence and requests for materials should be addressed to H.W. (email: hmwang@mail.sim.ac.cn) or to X.X. (email: xmxie@mail.sim.ac.cn).
} 
A $s$ the first isolated atomically thin crystal $^{1}$, graphene has attracted enormous interest from people all over the world because of its rich physical properties and great potential in various applications. Among them, applications in electronics are the most appealing, although they are challenging because they require high-quality, large-area samples ${ }^{2}$. Among the popular methods of graphene synthesis, chemical vapour deposition (CVD) is known as the most promising for scalable growth of high-quality graphene sheets ${ }^{3-18}$. Progress in this area, as demonstrated by the successful synthesis of 30 -inch continuous films ${ }^{19}$, the wafer-scale growth of a single-crystal monolayer of graphene on $\mathrm{Ge}^{20}$, and centimetre-sized graphene domains ${ }^{15}$, has been achieved. However, post-growth transfer remains a major hurdle for further applications because transfer processes introduce unavoidable surface/interface contamination, cracks and excessive wrinkles in graphene. Moreover, owing to the rough catalytic metal surface, the quality of the graphene grown is still far from the flatness of the mechanically exfoliated samples. Thus, the ability to develop a transfer-free technique by growing graphene directly on dielectric substrates with a large domain size, fast growth rate and very high quality is highly desirable.

Recently, molybdenum disulfide, tungsten disulfide and hexagonal boron nitride $(h-\mathrm{BN})$ are found to be good candidates for the substrate of graphene devices ${ }^{21}$. Among them, $h-\mathrm{BN}$ is obviously the best one because of its insulation properties, chemical inertness and small lattice misfit. Growing graphene directly on ultra-flat $h$-BN can greatly preserve the pristine properties of graphene, and the mobility of the aligned graphene grown on $h$-BN via the CVD method reaches $20,000-30,000 \mathrm{~cm}^{2} \mathrm{~V}^{-1} \mathrm{~s}^{-1}$ at room temperature ${ }^{22}$. However, this growth method suffers from the great drawback of very low growth rate $\left(\sim 1 \mathrm{~nm} \mathrm{~min}^{-1}\right)$ and the generation of domain sizes that are mostly smaller than $1 \mu \mathrm{m}$. Although the fabrication of a graphene domain $11 \mu \mathrm{m}$ in size has been achieved, it took $72 \mathrm{~h}$ to complete the fabrication ${ }^{23}$. Such a slow growth rate, which is three to four orders of magnitude slower than that obtained by CVD on a catalytic metal surface, originates mainly from the lack of a catalyst in the process.

In this report, we demonstrate that silane and germane as gaseous catalysts can boost the growth rate of graphene on $h$ - $\mathrm{BN}$ by almost two-order of magnitude higher than that in the absence of gaseous catalyst. The introduction of the gaseous catalyst also effectively improves the percentage of precisely aligned domains on $h$-BN. These results present a promising route towards very high-quality, transfer-free graphene on dielectric substrates for electronic applications.

\section{Results}

Gaseous catalyst assisted (GCA) graphene growth. A series of experiments are carried out to understand the effects of different gaseous catalysts upon the growth of graphene. Figure 1a presents the dependence of the domain size upon the growth time at a growth temperature of $1,280^{\circ} \mathrm{C}$. It is found that the domain size always increases linearly with growth time. Also, it is found that the growth rate of graphene in the absence of a catalyst is about $5 \mathrm{nmmin}-1$, which increases by an order of magnitude to $50 \mathrm{~nm} \mathrm{~min}^{-1}$ when germane is introduced. If silane is introduced, the growth rate further increases to $400 \mathrm{~nm} \mathrm{~min}^{-1}$ and, if the substrate is further heated to $1,350^{\circ} \mathrm{C}$, the growth rate reaches $\sim 1,000 \mathrm{~nm} \mathrm{~min}^{-1}$, which is very close to that of graphene grown on a metal surface by CVD (Fig. 1b). At such a high growth rate, the largest domain size of the graphene grown on $h$-BN reaches $20 \mu \mathrm{m}$ after $20 \mathrm{~min}$ of growth (Fig. 1b). As shown in Fig. $1 b^{7-15,24-27}$, using silane as a catalyst improves both the graphene growth rate and the typical domain size on $h$-BN by two orders of magnitude when compared with no catalyst. Auger electron spectroscopy is used to evaluate the amount of $\mathrm{Si}$ and $\mathrm{Ge}$ residue in the grown graphene samples (see Supplementary Fig. 4), whereupon no Si or Ge signal is detected, confirming that silane and germane are suitable to serve as catalysts for graphene growth. It is worthwhile to note that excessive supply of silane will cause formation of $\mathrm{SiC}$ nanoparticles (see Supplementary Fig. 3). The GCA-CVD method thereby bridges the huge gap between the direct growth of graphene on a dielectric substrate and that on a metal surface, and the graphene growth on $h$-BN presented here has already entered into the practical application regime. Figure 1c schematically illustrates the mechanism of graphene growth on $h$-BN, where the silicon atoms attach to the edge of the graphene domain and serve as the catalyst to reduce the reaction barrier for $\mathrm{C}_{2} \mathrm{H}_{2}$ molecules to form the honeycomb lattice along the graphene edge during growth.

Simulation of growth mechanism. To achieve a more detailed understanding of the role of silicon in graphene CVD growth, density functional theory (DFT) calculations are performed on armchair graphene, with the results shown in Fig. 2. Without a catalytic metal surface, the graphene growth front must be passivated by $\mathrm{H}$ atoms as the reaction is carried out in a
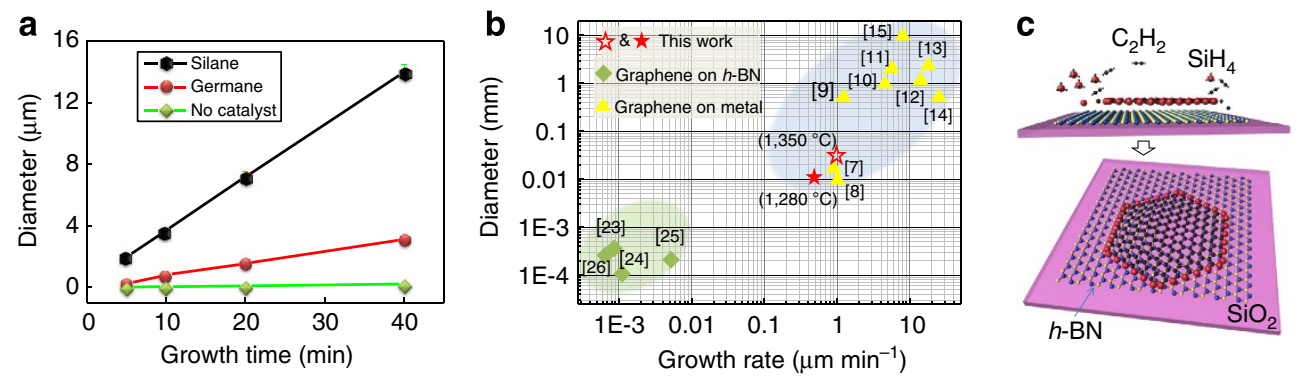

\footnotetext{
Figure 1 | The gaseous catalyst-assisted CVD of graphene on $\boldsymbol{h}$-BN. (a) The growth duration dependence of the domain dimensions for single-crystalline graphene in the presence of silane (black) or germane (red) gaseous catalysts and no catalyst (green). The diameter of the graphene domain is measured as the diagonal length of the hexagonal graphene crystal, and the growth temperature is $1,280^{\circ} \mathrm{C}$. (b) The graphene growth rates plotted as a function of the dimensions of the single-crystalline domains obtained in this work and those reported in the literature. The data points are labelled with the reference number from whence they came in brackets. (c) Schematic of the gaseous catalyst-assisted chemical vapour deposition process, visualizing the carbon (black), nitrogen (blue), boron (yellow), silicon (red) and hydrogen (small grey) atoms as spheres in the illustration. The schematic illustrates simplified schemes of the catalytic growth of monolayer graphene onto $h$ - $\mathrm{BN}$, where silicon atoms from the decomposition of $\mathrm{SiH}_{4}$ attach to the edge of the graphene and assist its growth.
} 

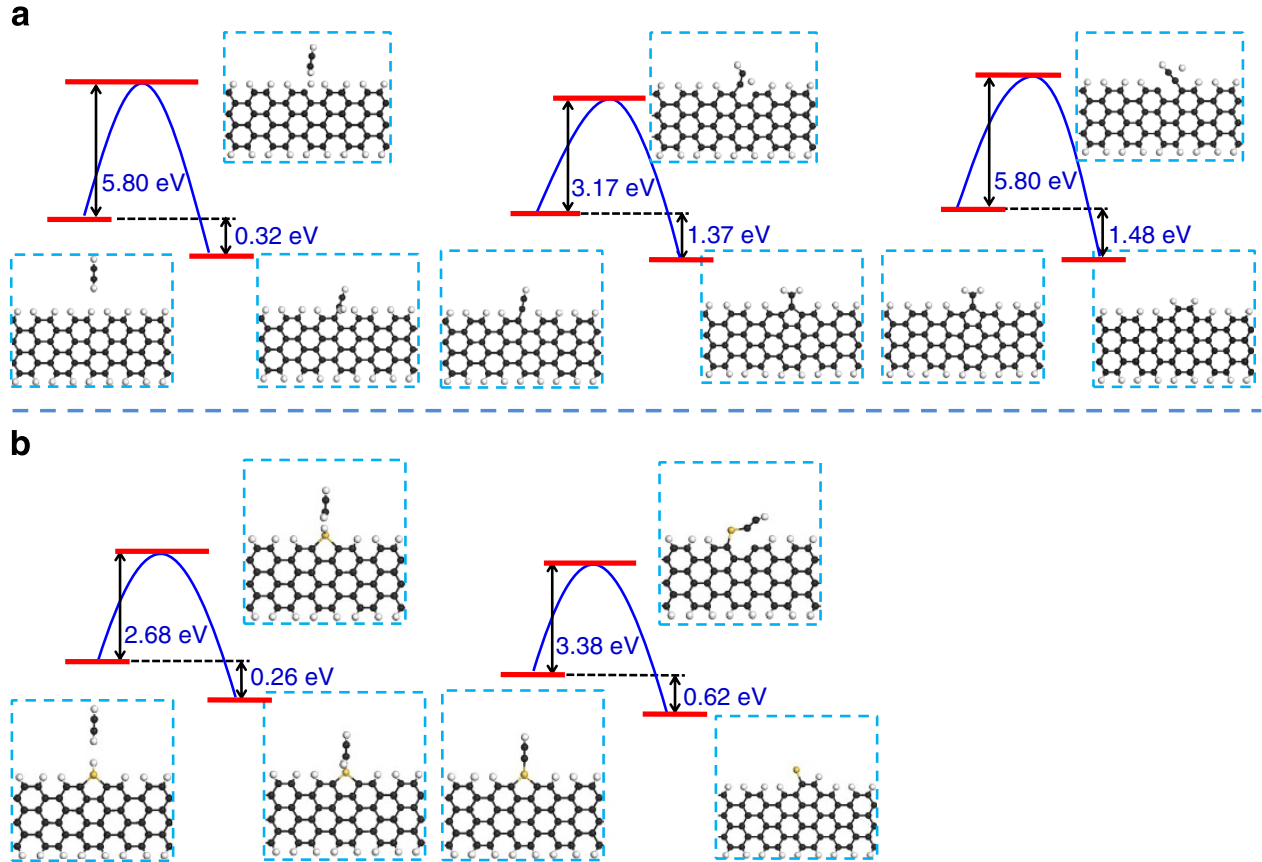

Figure 2 | Density functional calculation of the reaction between a $\mathrm{C}_{2} \mathrm{H}_{2}$ molecule with the commonly observed armchair graphene edge.

(a) without and (b) with an Si atom as a catalyst on the graphene edge. The black, white and yellow balls represent carbon, hydrogen and silicon atoms, respectively.
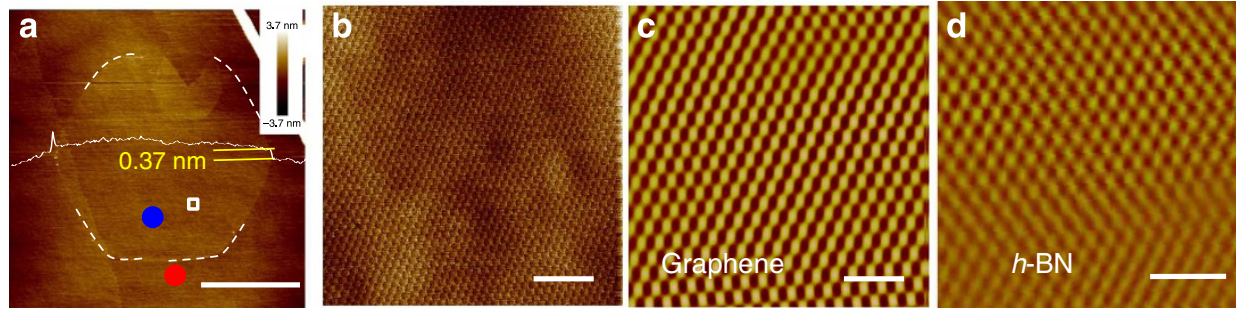

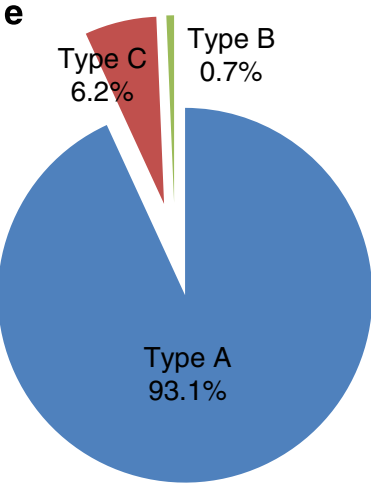

In the presence of silane

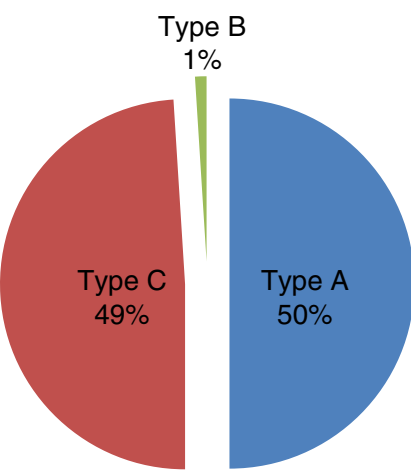

No gaseous catalyst

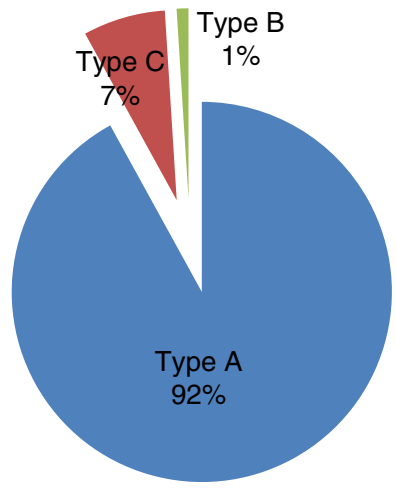

In the presence of germane

Figure 3 | The investigation on crystallinity and alignment of graphene domains grown on $\boldsymbol{h}$-BN. (a) Topography image of a typical single-crystalline graphene domain with a diameter of $20 \mu \mathrm{m}$. The dashed line frames the shape of the graphene grain. The line scan shows the graphene thickness to be $0.37 \mathrm{~nm}$. The scale bar is $10 \mu \mathrm{m}$. (b) The AFM friction image of the selected area in $\mathbf{a}$, where the giant moiré pattern with a periodicity of $13.9 \mathrm{~nm}$ can be clearly seen. The scale bar is $100 \mathrm{~nm}$. Atomic-resolution AFM images of (c) graphene and (d) $h$-BN taken from the areas marked by the blue and red dots in a, respectively. During the measurement, the scanning angles are always kept the same. The scale bars are $1 \mathrm{~nm}$. (e) Pie charts of the distribution of the type of graphene domains obtained with/without gaseous catalysts. Type ' $A$ ' indicates a graphene domain that is precisely aligned with the underlying $h$ - $\mathrm{BN}$, type ' $\mathrm{B}$ ' is one whose lattice is rotated $30^{\circ}$ with respect to the underlying $h$-BN lattice, and type ' $C$ ' is one with a polycrystalline structure.

hydrogen-rich environment. The chosen carbon feedstock, $\mathrm{C}_{2} \mathrm{H}_{2}$, ensures the formation of a complete hexagon for each cycle of $\mathrm{C}_{2} \mathrm{H}_{2}$ incorporation at the graphene edge. Our calculation indicates that three reaction steps are required to incorporate a $\mathrm{C}_{2} \mathrm{H}_{2}$ molecule onto the graphene edge to form a new hexagon, with corresponding energy barriers for these reaction steps of 5.80, 3.17 and $5.80 \mathrm{eV}$. In the case of a silicon catalyst (Fig. 2b), when a silicon atom attaches to the growth front, only two steps are 

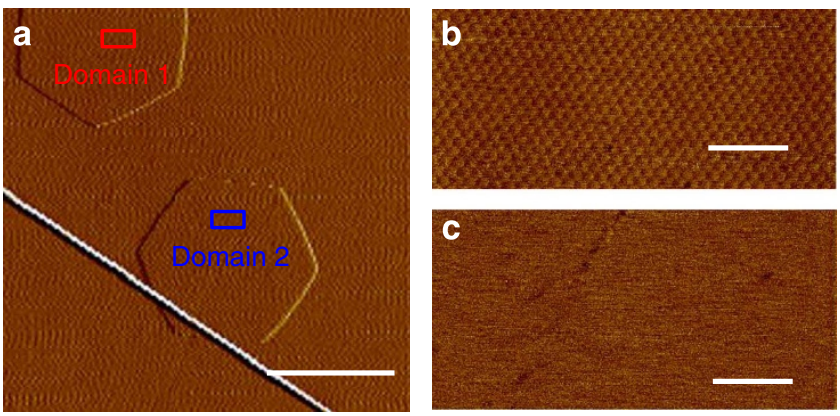

d

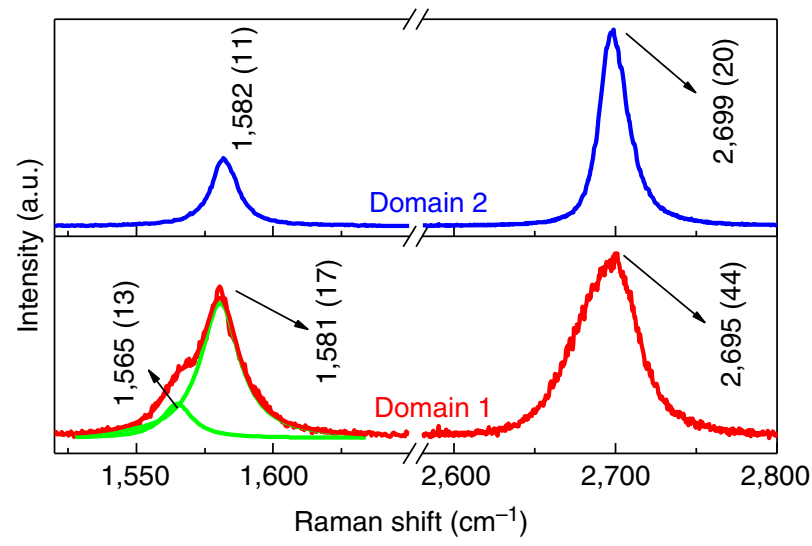

Figure 4 | Raman analyses on graphene domains grown on $\boldsymbol{h}$-BN.

(a) Topography images of two graphene domains grown on an $h-\mathrm{BN}$ surface. The white line across the image indicates a wrinkle on the $h-\mathrm{BN}$ surface formed during cooling. The scale bar is $2 \mu \mathrm{m}$. (b) Magnified view from the red box in $\mathbf{a}$, where the presence of a giant moire pattern indicates precisely aligned graphene with respect to the $h-\mathrm{BN}$. (c) Magnified view from the blue box in $\mathbf{a}$, where no detectable moire pattern is seen. The scale bars in $\mathbf{b}$ and $\mathbf{c}$ are $100 \mathrm{~nm}$. (d) Raman spectra taken from Domain 1 (lower plot) and Domain 2 (upper plot) in a. The full-width at half-maximum for each peak is given in parentheses with the peak location value, and the wavelength of the exciting laser is $488 \mathrm{~nm}$.

required to incorporate a $\mathrm{C}_{2} \mathrm{H}_{2}$ onto the graphene edge to form a new hexagon with corresponding energy barriers of 2.68 and $3.38 \mathrm{eV}$. Because each reaction step is exothermic, the threshold barriers for $\mathrm{C}_{2} \mathrm{H}_{2}$ incorporation onto a graphene edge without and with a $\mathrm{Si}$ atom as a catalyst are 5.80 and $3.38 \mathrm{eV}$, respectively. These calculations clearly indicate that this much-reduced threshold barrier when a catalyst is involved is responsible for the increased growth rate. Graphene edge activation by metal ad-atoms has been studied previously by simulations ${ }^{28-31}$. Experimentally, copper vapour was used in the demonstration of graphene growth on insulating substrates ${ }^{32,33}$. Oxygen was another surface activator, accelerating the graphene growth on $\mathrm{Cu}$ and suppressing its nucleation at the same time ${ }^{15}$. The use of silane/germane, conventional gases used in semiconductor industry with good accessibility and controllability, has not been previously proposed.

Atomic force microscopy characterization and alignment survey. The crystallinity and alignment of the graphene domains can be studied by analysing the moiré patterns obtained by atomic force microscopy (AFM), where the integrity of the pattern gives information of graphene crystallinity and the alignment can be judged by the periodicity and the rotation of the moire pattern with respect to the $h$-BN lattice ${ }^{22}$. M. Yankowitz et al. also did systematic investigations of the graphene/ $h$-BN super-lattice with different angular rotation ${ }^{34}$. In our experiments, three types of domains are observed. The 'A' domains present a regular hexagonal shape with a giant moiré pattern, whose edges are along the armchair direction. The periodicity of the moiré pattern is about $13.9 \mathrm{~nm}$, which demonstrates that these graphene domains are precisely aligned with the underlying $h-\mathrm{BN}^{22}$. The ' $\mathrm{B}$ ' domains also exhibit a regular hexagonal shape but with no detectable moiré patterns, and detailed atomic-resolution AFM measurements indicate that all of these ' $\mathrm{B}$ ' graphene domains are rotated $\sim 30^{\circ}$ with respect to the underlying $h$-BN lattice. The ' $\mathrm{C}$ ' domains exhibit the typical polycrystalline structure, with a detectable moiré pattern only on some sub-domains. Figure 3a shows a typical 'A' domain with a giant moiré pattern, shown in Fig. $3 \mathrm{~b}$, with the atomic-resolution image of the graphene and the underlying $h$-BN shown in Fig. $3 c$,d, respectively. The alignment of the moire pattern with respect to the $h$-BN is very sensitive to the angular separation between the graphene and $h$-BN when the angular separation is less than $1^{\circ}$ (ref. 22). Although the measurement may possess $\pm 3^{\circ}$ measurement error for the lattice orientation, mis-orientation between the graphene and $h$-BN should be less than $0.05^{\circ}$ by evaluating the alignment of the moire pattern with respect to the $h$-BN. These results prove that the graphene is precisely aligned with the underlying $h-\mathrm{BN}$. Details about the other two types of domains are given in Supplementary Figs 6 and 7 and Supplementary Discussion.

Figure $3 \mathrm{e}$ shows the statistics of the types of graphene domains grown with and without gaseous catalysts. In the absence of a gaseous catalyst, the precisely aligned domains (Type A) and polycrystalline domains (Type $\mathrm{C}$ ) appear in almost equal probability and only $\sim 1 \%$ domains are the $30^{\circ}$-rotated singlecrystalline domain (Type B). When using silane or germane as a catalyst, however, the percentage of precisely aligned domains (Type A) greatly improves to 93.1 or $92 \%$.

Raman characterization of graphene domains grown on $\boldsymbol{h}$-BN. Figure 4 shows the Raman spectra from Domain 1 (which is precisely aligned and noted as 'type A') and Domain 2 (type ' $\mathrm{B}$ ', which is a $30^{\circ}$-rotated single-crystalline domain). The Raman results for the aligned or misaligned graphene domains grown on $h$-BN by the GCA-CVD method are very similar to those for aligned or misaligned graphene flakes on $h$-BN made by mechanical exfoliation ${ }^{35}$, indicating that the GCA-CVD-grown graphene domains are of very high quality. It can be observed that the Raman spectrum of precisely aligned graphene exhibits obvious differences from that of rotated domains. In the precisely aligned graphene, the full-width at half-maximum (FWHM) of the $2 \mathrm{D}$ band is $\sim 44 \mathrm{~cm}^{-1}$, which is broader than that of graphene domains with a large misalignment angle. Moreover, a shoulder peak located at $1,565 \mathrm{~cm}^{-1}$ that is non-dispersive (see Supplementary Fig. 8) is noticeable, which can be attributed to a transverse optical phonon and may be activated by folding ${ }^{35,36}$. In addition, a more comprehensive analysis of the Raman results of the GCA-CVD-grown graphene domains is given in Supplementary Fig. 8 and Supplementary Table 2.

Electronic transport properties. A Hall bar device is made on a heavily doped silicon substrate for measuring the Hall and field effect mobilities (for details of the device fabrication, see Methods and Supplementary Fig. 9). Figure 5 shows the field effect characteristics of the device (Fig. 5a), the results of the Hall measurements at a temperature of $300 \mathrm{~K}$ and a magnetic field of $9 \mathrm{~T}$ (Fig. 5b), and the effects of Landau level splitting upon the longitudinal and Hall resistances at a temperature of $2 \mathrm{~K}$ (Fig $5 \mathrm{c}-\mathrm{d}$ ). The carrier-independent field effect mobility can be calculated from the plot in Fig. 5a, and is found to be $17,000 \mathrm{~cm}^{2} \mathrm{~V}^{-1} \mathrm{~s}^{-1}$ at $300 \mathrm{~K}$. The hole and electron mobilities can be derived 
a

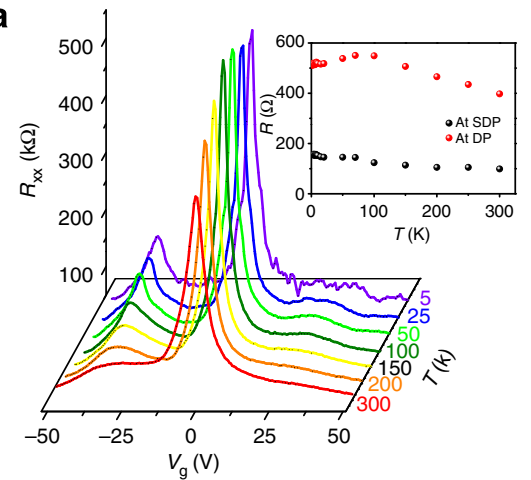

C

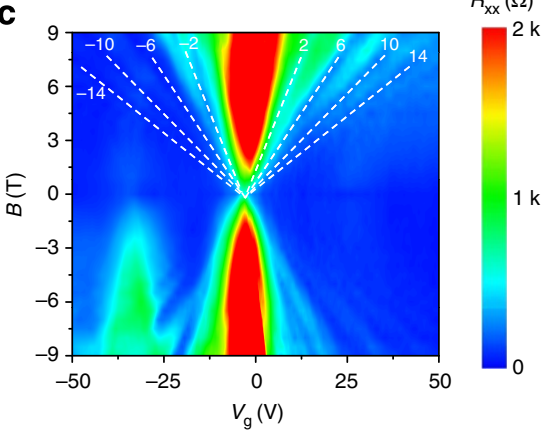

b
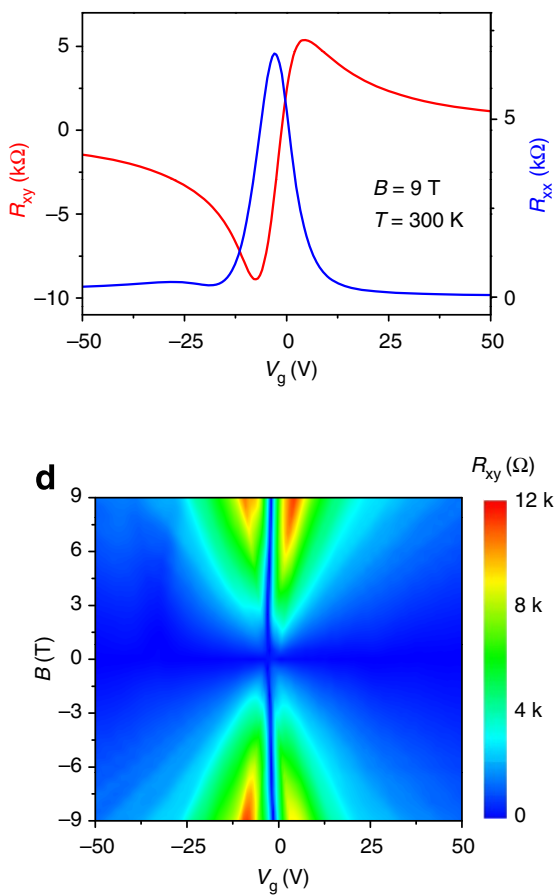

Figure 5 | Transport measurement of the single-crystalline graphene precisely aligned with the underlying $\boldsymbol{h}$-BN. (a) Back-gate voltage $\left(V_{g}\right)$ dependence of the longitudinal resistance at different temperatures. Inset shows the temperature dependence of the resistance at the Dirac point (DP; red spheres) and satellite peaks at the hole doping Secondary Dirac point (SDP) (black spheres). (b) Longitudinal ( $R_{\mathrm{xx}}$, blue) and Hall resistance ( $R_{\mathrm{xy}}$, red) versus $V_{\mathrm{g}}$ at temperature $T=300 \mathrm{~K}$ and magnetic field $B=9 \mathrm{~T}$. (c,d) Quantum Hall effect fan diagram of (c) $R_{\mathrm{xx}}$ and $(\mathbf{d}) R_{\mathrm{xy}}$ as a function of $V_{\mathrm{g}}$ and $B$ at a temperature of $2 \mathrm{~K}$.

from Fig. 5b, with values of 19,000 and $23,000 \mathrm{~cm}^{2} \mathrm{~V}^{-1} \mathrm{~s}^{-1}$, respectively. The high-mobility value measured for these samples indicates that the electrical quality of the graphene domains on $h$-BN is among the best for CVD-grown graphene $e^{15,37,38}$ and is comparable to that of micromechanically exfoliated graphene ${ }^{39}$ (see Supplementary Table 3).

Two side peaks are observed in Fig. 5a owing to secondary Dirac cones, which are a result of the graphene/h-BN moiré pattern, and are consistent with other reports ${ }^{40-42}$. It is worth noting that the resistances at the Dirac Point do not significantly increase with decreasing temperatures. Woods et al. have reported similar results, which in their study were explained as a result of the suppression of the commensurate state ${ }^{43}$.

In the plots of $R_{\mathrm{xx}}$ and $R_{\mathrm{xy}}$ as a function of both gate voltage and magnetic field, the standard Quantum Hall effect for graphene is observed to exhibit valleys in $R_{\mathrm{xx}}$ (Fig. 5c) and plateaus in $R_{\mathrm{xy}}$ (Fig. $5 \mathrm{~d}$ ) at the filling factors $v= \pm 4(n+1 / 2)$ $= \pm 2, \pm 6, \pm 10 \ldots$, where $n=0,1,2 \ldots$ is the Landau level index. A further investigation to demonstrate the effects of the secondary Dirac point upon the magnetic field is still underway.

\section{Discussion}

In summary, by combining the advantages of the catalytic CVD method and an ultra-flat dielectric substrate, we have developed a novel method to synthesize graphene domain sizes up to $20 \mu \mathrm{m}$ on $h$-BN with a growth rate comparable to that of graphene growth on metal surfaces and a graphene quality comparable to that made by the mechanical exfoliation method. In addition, the synthesized graphene on $h$-BN is transfer-free, which allows further electronic applications based on graphene/dielectric hetero-structures. Most of the graphene domains are perfectly aligned with the $h$-BN. No grain boundaries are observed when two domains merge; this brings hope to future fabrication of single crystalline graphene/h-BN wafer, which is essential for its scalable application on electronics.

\section{Methods}

Graphene synthesis. Before graphene growth, $h$-BN flakes were mechanically exfoliated onto quartz by Nitto tape and, after acetone cleaning, the quartz substrate with the $h$-BN flakes was loaded into the growth system. The graphene growth was carried out in a low-pressure CVD furnace at a temperature of $1,100-1,400{ }^{\circ} \mathrm{C}$. The system was then heated to $1,280^{\circ} \mathrm{C}$ under an $\mathrm{Ar} / \mathrm{H}_{2}(5: 1)$ mixture flow of $10 \mathrm{~cm}^{3}$ per min (s.c.c.m.), corresponding to $15 \mathrm{~Pa}$, and annealed for 5 min, after which the Ar/ $\mathrm{H}_{2}$ flow was turned off. The $\mathrm{C}_{2} \mathrm{H}_{2}$ flow and a mixture of silane/argon or germane/argon (5\% mole ratio of silane or germane to argon) were introduced into the system for the graphene growth. The pressure was kept at $5 \mathrm{~Pa}$ during the growth, and the growth time was in the range of 5-40 min. After growth, both the $\mathrm{C}_{2} \mathrm{H}_{2}$ and gaseous catalyst flow were turned off and the system was cooled down to room temperature with the $\mathrm{Ar} / \mathrm{H}_{2}$ mixture flowing. The samples of graphene on $h$-BN grown on a quartz surface were first transferred to a highly doped p-type silicon wafer with a 300-nm-thick $\mathrm{SiO}_{2}$ capping layer for electrical transport studies.

Scanning probe microscopy. As-grown samples were characterized by an AFM (Dimension Icon, Bruker) in contact mode, and the atomic-resolution images were obtained by the AFM (Multimode III, Veeco) under ambient conditions. The AFM images on different surfaces were recorded in contact mode using SNL-10 AFM tips from Bruker that possess a nominal tip radius of less than $10 \mathrm{~nm}$, and whose cantilevers possess a force constant $k=0.05-0.5 \mathrm{~N} \mathrm{~m}^{-1}$. To obtain a high accuracy, scanners with a travel range less than $10 \mu \mathrm{m}$ along the $X$ and $Y$ directions were used. Calibration for the atomic resolution was performed with newly cleaved highly ordered pyrolytic graphite (HOPG) before the measurements where, after calibration, the mean distance between the vicinal carbon atoms was measured to be $0.142 \mathrm{~nm}$. The integral gain and set-point were adjusted to be as low as possible to obtain optimal images, and the scan rate was set to a value in the range of $10-60 \mathrm{~Hz}$ to reduce the noise from thermal drift. Several hours of pre-scanning were carried out to warm up the scanner and ensure high imaging stability. Error in this orientation measurement varies by data points and was typically $\pm 2^{\circ}$, although it sometimes reached $\pm 3^{\circ}$.

DFT calculation. All of the calculations were performed within the framework of the DFT as implemented in the Vienna Ab-initio Simulation Package. 
The exchange-correlation potentials were treated by the gradient density approximation, and the interaction between valence electrons and ion cores was described by the projected augmented wave method. The energy cutoff for the plane wave functions was $400 \mathrm{eV}$ and the force acting on each atom was less than $0.02 \mathrm{eV} \AA^{-1}$. The energy barrier of every advance step was explored by climbing nudged elastic band method. The vacuum layer inside the super-cell was kept as large as $14 \AA$ to avoid interaction with the adjacent unit cell. The Brillion zone was sampled as $1 \times 2 \times 1$ grid meshes for the $7 \times 5$ slab and $1 \times 1 \times 1$ grid meshes for the larger $8 \times 6$ and $7 \times 8$ slabs using the Monkhorst-Pack scheme during the calculation.

Raman spectroscopy and mapping. Raman spectra were obtained with a WITec micro-Raman instrument possessing excitation laser lines of 488/532/633 nm. An objective lens of $\times 100$ magnification and a 0.95 numerical aperture was used, producing a laser spot that was $\sim 0.5 \mu \mathrm{m}$ in diameter. The laser power was kept less than $1 \mathrm{~mW}$ on the sample surface to avoid laser-induced heating. The Raman images were acquired using a WITec Raman system with a 600 lines/mm grating and a piezo crystal-controlled scanning stage under a $488-\mathrm{nm}$ laser excitation. The scanning step was about $200 \mathrm{~nm}$.

Device fabrication and electronic transport measurements. The Hall bar structure of the graphene devices was defined by a standard electron beam lithographic technique. The metal contacts $(60 \mathrm{~nm} \mathrm{Au} / 10 \mathrm{~nm} \mathrm{Ti})$ were deposited through electron beam evaporation, whereupon the devices were annealed in a hydrogen atmosphere at $250^{\circ} \mathrm{C}$ for $3 \mathrm{~h}$ to remove resist residues and to reduce the contact resistance between the graphene and electrodes before the electrical measurements. Because the thickness of the $h$-BN flake on the 300-nm-thick $\mathrm{SiO}_{2} / \mathrm{Si}$ substrates is about $20 \mathrm{~nm}$, the effective capacitance, $C_{\mathrm{g}}$, can be estimated as $10.5 \mathrm{nF} \mathrm{cm}^{-2}$. Both the electrical transport and magneto-transport measurements were carried out in a physical property measurement system (PPMS from Quantum Design, Inc.) via the standard lock-in technique.

\section{References}

1. Novoselov, K. S. et al. Electric field effect in atomically thin carbon films. Science 306, 666-669 (2004).

2. Novoselov, K. S. et al. A roadmap for graphene. Nature 490, 192-200 (2012).

3. Li, X. et al. Large-area synthesis of high-quality and uniform graphene films on copper foils. Science 324, 1312-1314 (2009).

4. Kim, K. S. et al. Large-scale pattern growth of graphene films for stretchable transparent electrodes. Nature 457, 706-710 (2009)

5. Robertson, A. W. \& Warner, J. H. Hexagonal single crystal domains of few-layer graphene on copper foils. Nano Lett. 11, 1182-1189 (2011).

6. Geng, D. et al. Uniform hexagonal graphene flakes and films grown on liquid copper surface. Proc. Natl Acad. Sci. USA 109, 7992-7996 (2012).

7. Wu, W. et al. Graphene: growth of single crystal graphene arrays by locally controlling nucleation on polycrystalline $\mathrm{Cu}$ using chemical vapor deposition. Adv. Mater. 23, 4898-4903 (2011)

8. Yu, Q. et al. Control and characterization of individual grains and grain boundaries in graphene grown by chemical vapour deposition. Nature Mater. 10, 443-449 (2011)

9. Li, X. et al. Large-area graphene single crystals grown by low-pressure chemical vapor deposition of methane on copper. J. Am. Chem. Soc. 133, 2816-2819 (2011).

10. Gao, L. et al. Repeated growth and bubbling transfer of graphene with millimetre-size single-crystal grains using platinum. Nat. Commun. 3, 699 (2012).

11. Chen, S. et al. Millimeter-size single-crystal graphene by suppressing evaporative loss of $\mathrm{Cu}$ during low pressure chemical vapor deposition. $A d v$. Mater. 25, 2062-2065 (2013).

12. Wu, T. et al. Triggering the continuous growth of graphene toward millimetersized grains. Adv. Funct. Mater. 23, 198-203 (2013).

13. Yan, Z. et al. Toward the synthesis of wafer-scale single-crystal graphene on copper foils. ACS Nano 6, 9110-9117 (2012).

14. Wang, H. et al. Controllable synthesis of submillimeter single-crystal monolayer graphene domains on copper foils by suppressing nucleation. J. Am. Chem. Soc. 134, 3627-3630 (2012).

15. Hao, Y. et al. The role of surface oxygen in the growth of large single-crystal graphene on copper. Science 342, 720-723 (2013).

16. Zhou, H. et al. Chemical vapour deposition growth of large single crystals of monolayer and bilayer graphene. Nat. Commun. 4, 2096 (2013).

17. Dai, B. et al. Rational design of a binary metal alloy for chemical vapour deposition growth of uniform single-layer graphene. Nat. Commun. 2, 522 (2011).

18. Pan, Y. et al. Highly ordered, millimeter-scale, continuous, single-crystalline graphene monolayer formed on Ru (0001). Adv. Mater. 21, 2777-2780 (2009).

19. Bae, S. et al. Roll-to-roll production of 30-inch graphene films for transparent electrodes. Nature Nanotech. 5, 574-578 (2010).

20. Lee, J. H. et al. Wafer-scale growth of single-crystal monolayer graphene on reusable hydrogen-terminated germanium. Science 344, 286-289 (2014).
21. Kretinin, A. V. et al. Electronic properties of graphene encapsulated with different two-dimensional atomic crystals. Nano Lett. 14, 3270-3276 (2014).

22. Tang, S. et al. Precisely aligned graphene grown on hexagonal boron nitride by catalyst free chemical vapor deposition. Sci. Rep. 3, 2666 (2013).

23. Chen, J. et al. Near-equilibrium chemical vapor deposition of high-quality single-crystal graphene directly on various dielectric substrates. Adv. Mater. 26, 1348-1353 (2013).

24. Yang, W. et al. Epitaxial growth of single-domain graphene on hexagonal boron nitride. Nature Mater. 12, 792-797 (2013).

25. Tang, S. et al. Nucleation and growth of single crystal graphene on hexagonal boron nitride. Carbon N. Y. 50, 329-331 (2012).

26. Son, M., Lim, H., Hong, M. \& Choi, H. C. Direct growth of graphene pad on exfoliated hexagonal boron nitride surface. Nanoscale 3, 3089-3093 (2011).

27. Garcia, J. M. et al. Graphene growth on h-BN by molecular beam epitaxy. Solid State Commun. 152, 975-978 (2012).

28. Raty, J., Gygi, F. \& Galli, G. Growth of carbon nanotubes on metal nanoparticles: a microscopic mechanism from ab initio molecular dynamics simulations. Phys. Rev. Lett. 95, 096103 (2005).

29. Liu, X. et al. Adsorption and growth morphology of rare-earth metals on graphene studied by ab initio calculations and scanning tunneling microscopy. Phys. Rev. B 82, 245408 (2010).

30. Gong, C., Colombo, L. \& Cho Photon-Assisted, K. CVD growth of graphene using metal adatoms as catalysts. J. Phys. Chem. C 116, 18263-18269 (2012).

31. Celebi, K. et al. Evolutionary kinetics of graphene formation on copper. Nano Lett. 13, 967-974 (2013).

32. Teng, P.-Y. et al. Remote catalyzation for direct formation of graphene layers on oxides. Nano Lett. 12, 1379-1384 (2012).

33. Kim, H. et al. Copper-vapor-assisted chemical vapor deposition for highquality and metal-free single-layer graphene on amorphous $\mathrm{SiO}_{2}$ substrate. ACS Nano 7, 6575-6582 (2013).

34. Yankowitz, M. et al. Emergence of superlattice dirac points in graphene on hexagonal boron nitride. Nature Phys. 8, 382-386 (2012).

35. Eckmann, A. et al. Raman fingerprint of aligned graphene/h-BN superlattices. Nano Lett. 13, 5242-5246 (2013).

36. Wang, Y. et al. Resonance Raman spectroscopy of G-line and folded phonons in twisted bilayer graphene with large rotation angles. Appl. Phys. Lett. 103, 123101 (2013).

37. Petrone, N. et al. Chemical vapor deposition-derived graphene with electrical performance of exfoliated graphene. Nano Lett. 12, 2751-2756 (2012).

38. Zhou, H. et al. Chemical vapour deposition growth of large single crystals of monolayer and bilayer graphene. Nat. Commun. 4, 2096 (2013).

39. Dean, C. R. et al. Boron nitride substrates for high-quality graphene electronics. Nature Nanotech. 5, 722-726 (2010).

40. Ponomarenko, L. A. et al. Cloning of Dirac fermions in graphene superlattices. Nature 497, 594-597 (2013)

41. Dean, C. R. et al. Hofstadter's butterfly and the fractal quantum Hall effect in moire superlattices. Nature 497, 598-602 (2013).

42. Hunt, B. et al. Massive dirac fermions and hofstadter butterfly in a van der Waals heterostructure. Science 340, 1427-1430 (2013).

43. Woods, C. R. et al. Commensurate-incommensurate transition for graphene on hexagonal boron nitride. Nature Phys. 10, 451-456 (2014).

\section{Acknowledgements}

We thank Y.B. Zhang from the Fudan University for fruitful discussions. The work at the Shanghai Institute of Microsystem and Information Technology, Chinese Academy of Sciences, is partially supported by the National Science and Technology Major Projects of China (grant no. 2011ZX02707), the Chinese Academy of Sciences (grant nos. KGZD-EW-303 and XDB04040300), and the projects from the Science and Technology Commission of Shanghai Municipality (grant nos. 12JC1410100 and 12JC1403900).

\section{Author contributions}

M.J. and X.X. directed the research work. H.W., X.X. and S.T. conceived and designed the experiments. S.T. fabricated the graphene samples. S.T. and H.W. performed the AFM experiments. H.W., H.X. and X.L. fabricated the electronic devices. H.W., H.S.W. and Q.S. performed the transport measurements. F.D., X.Y.Z., X.C. and X.H.Z. performed the DFT calculations. C.C. and T.Y. performed the Raman measurements. H.W., X.X., S.T., F.D., C.C. and T.Y. analysed the experimental data and designed the figures. H.W., S.T., C.C., T.Y., F.D., F.H., X.X. and M.J. co-wrote the manuscript and all authors contributed to critical discussions of the manuscript.

\section{Additional information}

Supplementary Information accompanies this paper at http://www.nature.com/ naturecommunications

Competing financial interests: The authors declare no competing financial interests. 
Reprints and permission information is available online at http://npg.nature.com/ reprintsandpermissions/

How to cite this article: Tang, S. et al. Silane-catalysed fast growth of large single-crystalline graphene on hexagonal boron nitride. Nat. Commun. 6:6499 doi: $10.1038 /$ ncomms7499 (2015). (c) (i) This work is licensed under a Creative Commons Attribution 4.0 International License. The images or other third party material in this article are included in the article's Creative Commons license, unless indicated otherwise in the credit line; if the material is not included under the Creative Commons license, users will need to obtain permission from the license holder to reproduce the material. To view a copy of this license, visit http://creativecommons.org/licenses/by/4.0/ 